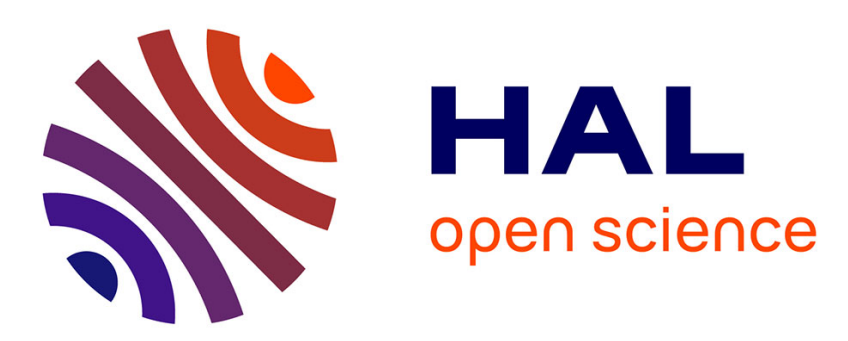

\title{
Transmission of time modulated optical signals through an absorbing photorefractive crystal
}

Philippe Delaye, Louis-Anne de Montmorillon, Gérald Roosen

\section{To cite this version:}

Philippe Delaye, Louis-Anne de Montmorillon, Gérald Roosen. Transmission of time modulated optical signals through an absorbing photorefractive crystal. Optics Communications, 1995, 118, pp.154-164. 10.1016/0030-4018(95)00169-9 . hal-00673981v2

\section{HAL Id: hal-00673981 \\ https://hal-iogs.archives-ouvertes.fr/hal-00673981v2}

Submitted on 30 Mar 2012

HAL is a multi-disciplinary open access archive for the deposit and dissemination of scientific research documents, whether they are published or not. The documents may come from teaching and research institutions in France or abroad, or from public or private research centers.
L'archive ouverte pluridisciplinaire HAL, est destinée au dépôt et à la diffusion de documents scientifiques de niveau recherche, publiés ou non, émanant des établissements d'enseignement et de recherche français ou étrangers, des laboratoires publics ou privés. 


\title{
TRANSMISSION OF TIME MODULATED OPTICAL SIGNALS THROUGH AN ABSORBING PHOTOREFRACTIVE CRYSTAL
}

\author{
Ph. Delaye, L.A. de Montmorillon, G.Roosen \\ Institut d'Optique Théorique et Appliquée, Unité de Recherche Associée 14 au Centre \\ National de la Recherche Scientifique, Bât.503, Centre Scientifique d'Orsay, B.P. 147, 91403 \\ Orsay Cedex, France, Email gerald.roosen@iota.u-psud.fr.
}

\begin{abstract}
$\underline{\text { Abstract }}$
We solve the problem of the transmission of time modulated signals in a two beam coupling photorefractive set-up using an absorbing material. We obtain an analytical expression that describes this phenomenon in absence of pump beam depletion due to beam coupling. We use this expression to analyse the influence of absorption on the photorefractive build-up. We also describe, using this model, the photorefractive beamsplitter response. It is also showed that the $\pi / 2$ phase shift between illumination and index gratings is detrimental to a linear detection of the phase variation of the signal beam.
\end{abstract}




\section{Introduction}

Coherent detection is a fundamental technique for the detection of phase modulated signals in which the coherent combination of the signal beam with a local oscillator requires a perfect adequacy of the wavefronts of the two beams. This is easily performed with guided waves in fiber optic communications, but, when considering free space communications, the problem is much more complex. The required phase matching of the signal and the local oscillator leads to a reduction of the field of view of the system, according to the antenna theorem [1]. However the use of photorefractive two-beam coupling allows to develop coherent detection schemes with large fields of view [2-4]. In this scheme, two beams, a pump beam and a time modulated signal beam, interfere in a photorefractive crystal. They create an index grating that is $\pi / 2$-shifted relative to the illumination grating. The beams then diffract on this grating, leading to an energy transfer from one beam to the other. Then, at the exit of the crystal, in the direction of the modulated signal beam, we have generation of a local oscillator which is the non modulated diffracted pump beam, having the same wavefront structure than the signal beam. This two beam coupling scheme is equivalent to the beam splitter in the classical homodyne detection scheme, but without the antenna theorem restriction as the signal and the local oscillator have the same phase structure whatever this structure. The photorefractive two beam coupling set-up then acts as a photorefractive beam splitter for homodyne detection [2,3]. An additional property of this component is its adaptability as the photorefractive effect is a dynamic effect. If the phase structure of the signal beam is slowly varying, the device will automatically adapt to this variation creating a new index structure. So the photorefractive beam splitter adapts to slow variations of the signal whereas it remains totally transparent to the rapidly modulated information imprinted on it.

This photorefractive beam splitter and its properties have been widely studied and used in the literature. Theories of operation of this component have been developed previously [2, 5-8]. Some of these models have taken into account the depletion of the pump beam due to the photorefractive energy transfer induced by high photorefractive gains $[7,9]$. However most of these models have considered that the influence of the crystal absorption on the kinetics was negligible, which is a strong and generally wrong assumption. Applications of this component in an industrial environment will require two important characteristics to be satisfied. First it should operate in the eye-safe part of the spectra, as for example the $1.5 \mu \mathrm{m}$ wavelength. Second, thanks to its adaptive properties, the component should be insensitive to environmental perturbations and should present a high cut-off frequency. A photorefractive response time of the order of $10 \mu \mathrm{s}$ is a good estimation of the required temporal performances [10]. First and second constraints impose the use of highly sensitive photorefractive 
semiconductors, like CdTe or GaAs [11,12]. These materials have low value electrooptic coefficients, which leads to low values of the photorefractive gain. The gains in these crystals are often of the order of magnitude or lower than the absorption. From these considerations, it is therefore not reasonable to consider that absorption is negligible, and we can not apply existing models without taking a lot of care. We then must solve the photorefractive two beam coupling model with a time varying signal taking absorption into account.

\section{Situation of the problem}

We send two plane waves on a photorefractive crystal, a strong unmodulated pump beam and a time varying signal beam (Fig.1). The signal can be either phase or amplitude modulated and has a much lower intensity than the pump beam. The aim of the study is to see how this modulation is transmitted through the crystal.

The photorefractive gain is supposed to be low, which means that the energy transfer is not high enough to induce depletion of the pump beam. We will be thus in the undepleted pump beam approximation. On the other hand, as we take into account absorption, the pump beam will be attenuated when traversing the crystal. Consequently the photorefractive time constant, that is determined by the pump beam intensity, will vary in the crystal thickness.

Pump beam and signal beam interfere in the crystal creating a spatially modulated space charge field. This space charge field induces, through the electrooptic effect, an index grating on which the beams diffract. This system is governed by three differential equations that link amplitude $E_{1}$ of the space charge field, amplitude $E_{i}$ of the pump beam and amplitude $\mathrm{E}_{\mathrm{d}}$ of the probe beam [5].

The first differential equation gives the kinetics of the space charge field [13] :

$$
\begin{aligned}
& \left.\left.-\left(\frac{\varepsilon k_{B} T}{I e^{2}}\right) \frac{\partial E_{1}}{\partial t}=E_{1}\left|\frac{A_{n} \alpha_{n}}{\kappa_{n}^{2}}\right| \frac{\frac{k(k-i V)}{k_{o}^{2}}+1}{\mid \frac{k(k-i V)}{\kappa_{n}^{2}}+1}\left|+\frac{A_{p} \alpha_{p}}{\kappa_{p}^{2}}\right| \frac{(k(k+i V)}{k_{o}^{2}}+1\right) \mid\right] \\
& +i m \frac{k_{B} T}{e}\left\{\frac{\alpha_{n}}{\kappa_{n}^{2}}\left|\frac{k-i V}{\mid \frac{k(k-i V)}{\kappa_{n}^{2}}+1}\right|-\frac{\alpha_{p}}{\kappa_{p}^{2}}\left|\frac{k+i V}{\mid \frac{k(k+i V)}{\kappa_{p}^{2}}+1}\right| \mid\right]
\end{aligned}
$$

Equation (1) can be rewritten as : 


$$
\frac{\partial E_{1}}{\partial t}+\frac{E_{1}}{\tau}=\frac{G}{\tau} \frac{E_{i}^{*} E_{d}}{I}
$$

with time constant $\tau$ of the space charge field (also labelled as photorefractive time constant) given by :

and

$$
\begin{aligned}
& \left.\frac{1}{\tau}=\frac{I e^{2}}{\varepsilon k_{B} T}\left|\frac{A_{n} \alpha_{n}}{\kappa_{n}^{2}}\right| \frac{\frac{k(k-i V)}{k_{o}^{2}}+1}{\mid \frac{k(k-i V)}{\kappa_{n}^{2}}+1}\left|+\frac{A_{p} \alpha_{p}}{\kappa_{p}^{2}}\right| \frac{\left.\frac{k(k+i V)}{k_{o}^{2}}+1\right)}{\frac{k(k+i V)}{\kappa_{p}^{2}}+1} \mid\right] \\
& \left.\frac{G}{\tau}=-2 i \frac{I e}{\varepsilon}\left|\frac{\alpha_{n}}{\kappa_{n}^{2}}\right| \frac{k-i V}{\mid \frac{k(k-i V)}{\kappa_{n}^{2}}+1}\left|-\frac{\alpha_{p}}{\kappa_{p}^{2}}\right| \frac{k+i V}{\mid \frac{k(k+i V)}{\kappa_{p}^{2}}+1}||\right]
\end{aligned}
$$

with $\quad m=2 \frac{E_{i}^{*} E_{d}}{I} \quad$ and $\quad I=E_{i}^{*} E_{i}+E_{d}^{*} E_{d}$

$\mathrm{m}$ is the modulation of the interference pattern and $\mathrm{I}$ is the total illumination (as we set $\mathrm{E}_{\mathrm{d}}<<\mathrm{E}_{\mathrm{i}}$, we have $\left.I=E_{i}^{*} E_{i}\right) . \mathrm{k}$ is the grating number ( $k=\frac{2 \pi}{\Lambda}$ with $\Lambda$ the grating spacing). In these expressions $\varepsilon$ is the dielectric constant of the material, $\alpha_{n}$ and $\alpha_{p}$ are the parts of absorption that generate electrons and holes respectively, $\kappa_{n}$ and $\kappa_{\mathrm{p}}$ are the inverse of the diffusion lengths of electrons and holes respectively, $\mathrm{k}_{0}$ is the inverse of the Debye screening length, $A_{n(p)}=\frac{S_{n(p)} I+\beta_{n(p)}}{S_{n(p)} I}$ with $S_{n(p)}$ the photoionisation cross-section of electrons (holes) and $\beta_{n(p)}$ the thermal emission coefficient of electrons (holes), $V=\frac{e E_{0}}{k_{B} T}$ with $\mathrm{E}_{0}$ the applied external electric field.

If we neglect the influence of $\mathrm{A}_{\mathrm{n}}$ and $\mathrm{A}_{\mathrm{p}}$ (i.e. photoconductivity much larger than dark conductivity) we have $\frac{1}{\tau}$ proportional to I and $\frac{G}{\tau}$ proportional to I, which means that $\mathrm{G}$ is independent of I. In the general case, $\mathrm{G}$ and $\frac{1}{\tau}$ are complex. In the particular case of a photorefractive effect governed by the diffusion regime $\left(E_{0}=0\right), \frac{1}{\tau}$ is real and $G$ is purely imaginary.

The two other differential equations are the propagation equations for the pump and signal beams [14] :

$$
\left\{\begin{array}{l}
\frac{\partial E_{i}}{\partial x}=\frac{\pi n_{0}^{3} r_{e f f} m^{*} E_{s c}^{*}}{2 i \lambda \cos \theta} E_{d} \\
\frac{\partial E_{d}}{\partial x}=\frac{\pi n_{0}^{3} r_{e f f} m E_{s c}}{2 i \lambda \cos \theta} E_{i}
\end{array}\right.
$$


$r_{\text {eff }}$ is the effective electrooptic coefficient that depends on the orientation of the photorefractive crystal and on the directions of polarization of the beams; $n_{0}$ is the linear index of refraction of the material, $\lambda$ is the wavelength in the material, $\theta$ is the half-angle between the beams inside the material and the beams propagate along the $\mathrm{x}$ direction.

We set $\mathrm{E}_{1}=m \mathrm{E}_{s c}$ and $\frac{\gamma}{G}=\frac{\pi n_{0}^{3} r_{\text {eff }}}{2 i \lambda \cos \theta}$ which gives $\gamma=\frac{\pi n_{0}^{3} r_{\text {eff }}}{2 i \lambda \cos \theta} G$. Here we can note that in the diffusion regime (without any applied electric field) $\gamma$ is purely real and equals $\gamma=\frac{\Gamma}{2}$ with $\Gamma$ the two beam coupling photorefractive intensity gain $\left(\Gamma\right.$ in $\left.\mathrm{cm}^{-1}\right)[13,14]$.

Taking into account all these notations and including the absorption ( $\alpha$ absorption coefficient for intensity in $\mathrm{cm}^{-1}$ ) equations (3) become :

$$
\left\{\begin{array}{l}
\frac{\partial E_{i}}{\partial x}=\frac{\gamma}{G} E_{1}^{*} E_{d}-\frac{\alpha}{2} E_{i} \\
\frac{\partial E_{d}}{\partial x}=\frac{\gamma}{G} E_{1} E_{i}-\frac{\alpha}{2} E_{d}
\end{array}\right.
$$

(2) and (4) correspond to a system of three coupled differential equations.

In this problem we are interested in the transmission through the crystal of the time modulated signal beam $E_{d}$. We will rewrite these three equations in the form of one partial differential equation that governs the propagation of $E_{d}$ through the crystal. For this resolution we will make some assumptions and approximations, we will now precise.

1.- The pump beam is not time modulated (an eventual phase modulation present on the pump beam can be easily transferred on the signal beam as it is the phase difference between the two beams that is important).

2.- We neglect the pump beam depletion due to the beam coupling, it corresponds to a regime of small photorefractive gain and high pump to probe beam ratio.

3.- The pump beam attenuation due to the absorption is taken into account.

4.- At time $\mathrm{t}=0$ (before the probe beam is modulated) we are in a steady-state regime with a grating written in the crystal. It means that before $t=0$, the two beams are present in the crystal and have written a saturated index grating on which they now diffracts (if no grating is present before $\mathrm{t}=0$, the system can be solved as well, as it will be seen later).

Points 2 and 3 allow to simplify equation (4a) that becomes :

$$
\frac{\partial E_{i}}{\partial x}=-\frac{\alpha}{2} E_{i}
$$

It is solved immediately as : $E_{i}(x)=E_{i 0} e^{-\frac{\alpha}{2} x} \quad$ independent of time.

It finally gives the total illumination in the crystal : $I(x)=E_{i} E_{i}^{*}+E_{d} E_{d}^{*}=E_{i} E_{i}^{*}=I_{0} e^{-\alpha x}$ with $I_{0}=\left|E_{i 0}\right|^{2}$ the pump beam intensity at the entrance of the crystal. 
We see from this first equation that the illumination decreases during propagation through the crystal. Then the time constant $\tau$ increases during this same propagation (the photorefractive effect is slower at the exit of the crystal than at its entrance). As $\frac{1}{\tau}$ is proportional to I, we can write $\frac{1}{\tau}=\frac{e^{-\alpha x}}{\tau_{0}}$ with $\tau_{0}$ the photorefractive time constant at the entrance of the crystal (at $\mathrm{x}=0)$.

Deriving equation (4b) relative to time $\mathrm{t}$ and replacing $\frac{\partial E_{1}}{\partial t}$ by its expression given by equation (2) we obtain :

$$
\frac{\partial^{2} E_{d}}{\partial t \partial x}=\frac{\gamma}{\tau} \frac{E_{i} E_{i}^{*}}{I} E_{d}-\frac{\gamma}{G \tau} E_{1} E_{i}-\frac{\alpha}{2} \frac{\partial E_{d}}{\partial t}
$$

We then take $\mathrm{E}_{1}$ from equation (4b) which finally gives the partial derivative equation that governs $E_{d}(x, t)$ :

$$
\frac{\partial^{2} E_{d}}{\partial t \partial x}=\frac{E_{d} e^{-\alpha x}}{\tau_{0}}\left(\gamma-\frac{\alpha}{2}\right)-\frac{e^{-\alpha x}}{\tau_{0}} \frac{\partial E_{d}}{\partial x}-\frac{\alpha}{2} \frac{\partial E_{d}}{\partial t}
$$

Point 4 gives us the initial condition of the problem. At $\mathrm{t}=0$, a grating is written in the crystal and is in its stationary state, so we have : $E_{d}(x, 0)=E_{d 0} e^{-\frac{\alpha}{2} x} e^{\gamma x}$. At time $\mathrm{t}=0^{+}$, we put a known time modulation (in amplitude and/or in phase) on the signal beam $E_{d}(0, t)$ at the entrance of the crystal and we are looking for the expression of $E_{d}(x, t)$ after propagation of a distance $\mathrm{x}$ inside the crystal.

In order to simplify the equation we will change the function by setting $E_{d}(x, t)=F(x, t) e^{\gamma x} e^{-\frac{\alpha x}{2}}$. Equation (7) becomes :

$$
\frac{\partial^{2} F}{\partial t \partial x}=-\gamma \frac{\partial F}{\partial t}-\frac{e^{-\alpha x}}{\tau_{0}} \frac{\partial F}{\partial x}
$$

with the initial condition at $\mathrm{t}=0: F(x, 0)=E_{d}(0,0)$ constant, which means that $\frac{\partial F}{\partial x}(x, 0)=0$. The boundary condition $F(0, t)=E_{d}(0, t)$ is already known.

\section{Resolution of the transfer equation}

We will solve equation (8) using the Laplace transform method. The Laplace transform method is prefered over the Fourier transform method [15, 16] as it deals more easily with the causal signals we have in our study. We call $\tilde{F}(x, s)$ the Laplace transform of $F(x, t)$, with the initial conditions $F(x, 0)=E_{d}(0,0), \frac{\partial F}{\partial x}(x, 0)=0$ and the boundary 
condition that becomes $\tilde{F}(0, s)$ (Laplace transform of $F(0, t))$ known. We take the Laplace transform of equation (8) which gives (following functions with tilde are functions of s while functions without tilde are functions of $\mathrm{t}$ ) :

$$
\left(s+\frac{1}{\tau_{0} e^{\alpha x}}\right) \frac{\partial \tilde{F}}{\partial x}=-\gamma s \tilde{F}+\gamma E_{d}(0,0)
$$

which is rewritten :

$$
\frac{\partial \tilde{F}}{\partial x}(x, s)=-\frac{\gamma s \tau_{0} e^{\alpha x}}{1+s \tau_{0} e^{\alpha x}} \tilde{F}(x, s)+\frac{\gamma \tau_{0} e^{\alpha x}}{1+s \tau_{0} e^{\alpha x}} E_{d}(0,0)
$$

The solution of this differential equation (function of $\mathrm{x}$ ) is :

$$
\tilde{F}(x, s)=C(s)\left(\frac{1+s \tau_{0}}{1+s \tau_{0} e^{\alpha x}}\right)^{\frac{\gamma}{\alpha}}+\frac{E_{d}(0,0)}{s}
$$

The integration constant $\mathrm{C}(\mathrm{s})$ is given by the boundary condition $: \tilde{F}(0, s)=C(s)+\frac{E_{d}(0,0)}{s}$.

Going back to $\tilde{E}_{d}(x, s)$ the Laplace transform of $E_{d}(x, t)$, with $\tilde{F}(0, s)=\tilde{E}_{d}(0, s)$, we obtain finally :

$$
\tilde{E}_{d}(x, s)=\left[\tilde{E}_{d}(0, s)-\frac{E_{d}(0,0)}{s}\right] e^{-\frac{\alpha x}{2}} e^{\gamma x}\left(\frac{1+s \tau_{0}}{1+s \tau_{0} e^{\alpha x}}\right)^{\frac{\gamma}{\alpha}}+\frac{E_{d}(0,0)}{s} e^{-\frac{\alpha x}{2}} e^{\gamma x}
$$

The solution $E_{d}(x, t)$ is given by the inverse Laplace transform of expression (12).

In order to find $E_{d}(x, t)$, we set $\tilde{H}(x, s)=\left(\frac{1}{1+s \tau_{0}}\right)\left(\frac{1+s \tau_{0}}{1+s \tau_{0} e^{\alpha x}}\right)^{\frac{\gamma}{\alpha}}$, which inverse Laplace transform is $H(x, t)$. Equation (12) becomes :

$$
\tilde{E}_{d}(x, s)=e^{-\frac{\alpha x}{2}} e^{\gamma x}\left[\left[\tilde{E}_{d}(0, s)-\frac{E_{d}(0,0)}{s}\right]\left(1+s \tau_{0}\right) \tilde{H}(x, s)+\frac{E_{d}(0,0)}{s}\right]
$$

which gives :

$$
\tilde{E}_{d}(x, s)=e^{-\frac{\alpha x}{2}} e^{\gamma x}\left[\left[\tilde{E}_{d}(0, s)-\frac{E_{d}(0,0)}{s}\right] \tilde{H}(x, s)+\tau_{0}\left[s \tilde{E}_{d}(0, s)-E_{d}(0,0)\right] \tilde{H}(x, s)+\frac{E_{d}(0,0)}{s}\right]
$$

We then take the inverse Laplace transform of expression (14), using the convolution theorem, and we obtain the final expression for $E_{d}(x, t)$ :

$$
E_{d}(x, t)=e^{-\frac{\alpha x}{2}} e^{\gamma x}\left[E_{d}(0,0)+\int_{0}^{t}\left(\tau_{0} \frac{\partial E_{d}}{\partial t}(0, T)+E_{d}(0, T)-E_{d}(0,0)\right) H(x, t-T) d T\right]
$$

We thus have an analytical expression for the transmission of the modulated signal through a photorefractive crystal in a two beam coupling configuration. The problem is now to find the expression of $H(x, t)$. 
In a general way, we are looking for the inverse Laplace transform of function $\tilde{H}(s)=\frac{(1+a s)^{\lambda-1}}{(1+b s)^{\lambda}}$.

We rewrite this function as $\tilde{H}(s)=\frac{a^{\lambda-1}}{b^{\lambda}} \tilde{F}\left(s+\frac{1}{a}\right)$ with $\tilde{F}(s)=\frac{s^{\lambda-1}}{\left(s-\frac{b-a}{a b}\right)^{\lambda}}$ function which inverse Laplace transform is known from tables [17]. This function is ${ }_{1} \mathrm{~F}_{1}\left(\lambda, 1,\left(\frac{b-a}{a b}\right) t\right)$ with the confluent hypergeometric function ${ }_{1} \mathrm{~F}_{1}(a, b, z)$ [18]. We then deduce, knowing the properties of the Laplace transform, that the inverse Laplace transform of $\tilde{H}(s)$ is $H(t)=\frac{a^{\lambda-1}}{b^{\lambda}} e^{-\frac{t}{a}}{ }_{1} \mathrm{~F}_{1}\left(\lambda, 1,\left(\frac{b-a}{a b}\right) t\right)$. In our case of interest, i.e. $a=\tau_{0}, b=\tau_{0} e^{\alpha x}$ and $\lambda=\frac{\gamma}{\alpha}$, we finally obtain :

$$
H(x, t)=\frac{e^{-\gamma x}}{\tau_{0}} e^{-\frac{t}{\tau_{0}}}{ }_{1} \mathrm{~F}_{1}\left(\frac{\gamma}{\alpha}, 1,\left(\frac{e^{\alpha x}-1}{e^{\alpha x}}\right) \frac{t}{\tau_{0}}\right)
$$

So this value of function $H(x, t)$ completely determines the value of $E_{d}(x, t)$ given by relation (15).

We will now show that the expression we find, reduces, in the case of no absorption, to the expression previously derived in the literature [5].

For negligible absorption $\alpha$ goes to 0 , and then $\lambda=\frac{\gamma}{\alpha}$ goes to infinity. Setting $z=-\left(\frac{e^{\alpha x}-1}{e^{\alpha x}}\right)\left(\frac{-t \gamma}{\alpha \tau_{0}}\right) \underset{\alpha \rightarrow 0}{\longrightarrow}-\frac{\gamma x t}{\tau_{0}}$, we rewrite ${ }_{1} \mathrm{~F}_{1}\left(\frac{\gamma}{\alpha}, 1,\left(\frac{e^{\alpha x}-1}{e^{\alpha x}}\right) \frac{t}{\tau_{0}}\right)={ }_{1} \mathrm{~F}_{1}\left(\frac{\gamma}{\alpha}, 1,-z \frac{\alpha}{\gamma}\right)$. As we know that $\lim _{a \rightarrow+\infty} \mathrm{F}_{1}\left(a, 1,-\frac{z}{a}\right)=\mathrm{J}_{0}(2 \sqrt{z}) \quad[18]$, we finally deduce that $\lim _{\alpha \rightarrow 0}{ }_{1} \mathrm{~F}_{1}\left(\frac{\gamma}{\alpha}, 1,\left(\frac{e^{\alpha x}-1}{e^{\alpha x}}\right) \frac{t}{\tau_{0}}\right)=\mathrm{J}_{0}\left(2 \sqrt{-\frac{\gamma x t}{\tau_{0}}}\right)$

and then :

$$
\lim _{\alpha \rightarrow 0} H(x, t)=\frac{e^{-\gamma x}}{\tau_{0}} e^{-\frac{t}{\tau_{0}}} \mathbf{J}_{0}\left(2 \sqrt{-\frac{\gamma x t}{\tau_{0}}}\right)
$$

To be exactly in the case established by Cronin-Golomb [5], the initial conditions have to be changed a little and we have to consider that no grating is present at time $\mathrm{t}=0$. In that case we would have to slightly modify function $F(x, t)$, defining it as $E_{d}(x, t)=F(x, t) e^{-\frac{\alpha x}{2}}$ with the initial conditions $F(x, 0)=E_{d}(0,0)$ and $\frac{\partial F}{\partial x}(x, 0)=0$. The equation would become :

$$
\frac{\partial^{2} F}{\partial t \partial x}=\gamma \frac{e^{-\alpha x}}{\tau_{0}} F-\frac{e^{-\alpha x}}{\tau_{0}} \frac{\partial F}{\partial x}
$$


The same resolution as for the previous equations would then have given :

$$
E_{d}(x, t)=e^{-\frac{\alpha x}{2}} e^{\gamma x}\left\lfloor\tau_{0} E_{d}(0,0) H(x, t)+\int_{0}^{t}\left(\tau_{0} \frac{\partial E_{d}}{\partial t}(0, T)+E_{d}(0, T)\right) H(x, t-T) d T\right\rfloor
$$

with the same function $\mathrm{H}(\mathrm{x}, \mathrm{t})$.

So when $\alpha$ goes to 0 considering expression (17), equation (19) reduces to :

$$
E_{d}(x, t)=E_{d}(0,0) K(t)+\int_{0}^{t}\left(\frac{\partial E_{d}}{\partial t}(0, T)+\frac{E_{d}(0, T)}{\tau_{0}}\right) K(t-T) d T
$$

with $K(t)=e^{-\frac{t}{\tau_{0}}} \mathbf{J}_{0}\left(2 \sqrt{-\frac{\gamma x t}{\tau_{0}}}\right)$. This expression is the one derived in Ref.[5] after correction of a typographical error [6].

So our model is a generalization of the model of Cronin-Golomb to the case where absorption is not negligible, which is the real case.

We have established an analytical expression (expressions (15) and (16)) of the transmission of a time varying signal through an absorbing photorefractive crystal in a two beam coupling scheme. The final expression has the same feature than in the case without absorption, the only difference being in the transfer function which is a less common function than the Bessel function found by Cronin-Golomb. We have transformed the problem from solving a system of coupled partial derivative equations to the one of solving an expression with still a convolution but with one variable $t$ only. The expression is still complicated even if easy to solve numerically. In fact, in some special cases this expression can be simplified. We will now present two of these cases.

\section{Two-beam coupling build-up}

An example of the use of the expression we have established, is for the determination of the build-up kinetics of the two beam coupling effect in presence of absorption. Absorption creates a continuum of time constants through out the crystal as the illumination decreases due to absorption. The question is then, what is the actual kinetics of energy transfer ?

To solve the problem we use expression (19) with a step function for the initial value : $E_{d}(0, t)=0$ for $t \leq 0$ and $E_{d}(0, t)=1$ for $t>0$. The derivative of this function is a Dirac function centered at 0 . We have then :

$$
E_{d}(x, t)=e^{-\frac{\alpha x}{2}} e^{\gamma x}\left\lceil\tau_{0} H(x, t)+\int_{0}^{t} H(x, t-T) d T\right]=e^{-\frac{\alpha x}{2}} e^{\gamma x}\left\lceil\tau_{0} H(x, t)+\int_{0}^{t} H(x, T) d T\right\rfloor
$$


When absorption is negligible we replace $H(x, t)$ by function $K(t)$ and we obtain the expression derived in Ref.[19].

From the analytical expression (21), we have numerically calculated the temporal dependency of the output intensity, for a crystal with an intensity gain of $\Gamma=0.3 \mathrm{~cm}^{-1}$, a thickness $\mathrm{x}=1 \mathrm{~cm}$ and a time constant $\tau_{0}=1 \mathrm{~s}$, for different values of absorption between 0 and $1 \mathrm{~cm}^{-1}$. Results are shown in Fig. 2 where the different curves were normalized to their steady state value : $S=\left|\frac{E_{d}(x, t)}{e^{-\frac{\alpha x}{2}} e^{\gamma x}}\right|^{2}$. We see on these curves that the more absorbent the crystal, the slower the two beam coupling build-up. Moreover these kinetics curves are very close to exponential curves with two beam coupling build-up time constants that follows the law $\tau_{T B C}(\alpha)=e^{\frac{\alpha x}{2}} \tau_{T B C}(\alpha=0)$ which corresponds to a time constant value taken in the middle of the crystal. We here have to note that, in our simulations, we have neglected a contribution of the absorption on the photorefractive effect. Indeed space charge field time constant $\tau_{0}$ depends directly on the absorption of the crystal (through parameters $\alpha_{n}$ and $\alpha_{p}$ in relation (2a)). In the calculations we only wanted to show the influence of absorption on two beam coupling, so we take the same value of time constant $\tau_{0}$ for all curves, despite the fact that, in real cases, changing absorption changes the response time of the space charge field build-up.

We also note on the curve at $\alpha=0$ that the exponential time constant is greater than $\tau_{0}$ as already noticed in Ref.[9]. This means that the build-up of the space charge field takes a longer time in presence of beam coupling than when beam coupling is absent (when for example $r_{\text {eff }}=0$ ). This slowing down of the kinetics with increasing beam coupling gain can be seen more clearly in Fig. 3 where we have calculated the normalized transmitted probe beam intensity for different values of the photorefractive gain between 0 and $2 \mathrm{~cm}^{-1}$. The grating build-up slowers as the photorefractive gain increases.

\section{Detection of rapidly phase modulated signals : Photorefractive beamsplitter}

One use of the photorefractive beamsplitter is the detection of rapidly phase modulated signals $[4,20]$. We want to measured a signal $E_{d}(0, t)$ which is phase modulated, on a time scale very short compared to the response time of the photorefractive effect $\tau_{0}$. We are then in experimental conditions where $\mathrm{t}<<\tau_{0}$. As we have $\lim _{z \rightarrow 0}{ }_{1} \mathrm{~F}_{1}(a, b, z)=1$, equation (15) becomes :

$$
E_{d}(x, t)=e^{-\frac{\alpha x}{2}} e^{\gamma x}\left[E_{d}(0,0)+\int_{0}^{t}\left(\tau_{0} \frac{\partial E_{d}}{\partial t}(0, T)+E_{d}(0, T)-E_{d}(0,0)\right) \frac{e^{-\gamma x}}{\tau_{0}} e^{-\frac{t-T}{\tau_{0}}} d T\right]
$$


which is rewritten as :

$$
E_{d}(x, t)=e^{-\frac{\alpha x}{2}}\left[E_{d}(0,0) e^{\gamma x}+e^{-\frac{t}{\tau_{0}} t} \int_{0}\left(\frac{\partial}{\partial T}\left[e^{\frac{T}{\tau_{0}}}\left(E_{d}(0, T)-E_{d}(0,0)\right)\right]\right) d T\right\rfloor
$$

We have finally :

$$
E_{d}(x, t)=e^{-\frac{\alpha x}{2}}\left[E_{d}(0,0) e^{\gamma x}+\left(E_{d}(0, t)-E_{d}(0,0)\right)\right]
$$

Knowing that $E_{d}(x, 0)=E_{d}(0,0) e^{\gamma x} e^{-\frac{\alpha x}{2}}$, we obtain the simple expression that gives the transmission of the modulated signal through the crystal (when a stationary regime is reached)

$$
\left(E_{d}(x, t)-E_{d}(x, 0)\right)=e^{-\frac{\alpha x}{2}}\left(E_{d}(0, t)-E_{d}(0,0)\right)
$$

The modulated input signal $E_{d}(0, t)$ can be written as $E_{d}(0, t)=E_{d}(0,0) \times \Delta E_{d}(t)$ with $\Delta E_{d}(0)=1$. It then gives for relation (24) :

$$
E_{d}(x, t)=e^{-\frac{\alpha x}{2}} E_{d}(0,0)\left[\left(e^{\gamma x}-1\right)+\Delta E_{d}(t)\right]
$$

Experimentally, we are interested in the intensity of the signal beam at the output of the crystal. It is given by :

$$
I_{d}(x, t)=e^{-\alpha x} I_{d}(0,0)\left[\left|e^{\gamma x}-1\right|^{2}+\left|\Delta E_{d}(t)\right|^{2}+2 \operatorname{Re}\left(\left(e^{\gamma x}-1\right)^{*} \Delta E_{d}(t)\right)\right]
$$

The signal beam is now purely phase modulated, so we have $\Delta E_{d}(t)=e^{i \varphi(t)}$ with $\varphi(0)=0$, what gives $\left|\Delta E_{d}(t)\right|^{2}=1$. We write the photorefractive gain as $\gamma=\gamma^{\prime}+i \gamma^{\prime \prime}$. The gain is purely real, $\gamma^{\prime \prime}=0$, when the phase shift between index and illumination gratings is $\pi / 2$ (photorefractive effect in the diffusion regime) and is purely imaginary, $\gamma^{\prime}=0$, when the two gratings are in phase. According to these notations, the rapidly modulated part of the transmitted pump intensity $\Delta I_{d}(x, t)$ is due to the third term of expression (27) only and we finally have :

$$
\Delta I_{d}(x, t)=2 e^{-\alpha x} I_{d}(0,0)\left[e^{\gamma^{\prime} x} \sin \left(\gamma^{\prime \prime} x\right) \sin (\varphi(t))+\left(e^{\gamma^{\prime} x} \cos \left(\gamma^{\prime \prime} x\right)-1\right) \cos (\varphi(t))\right]
$$

In this expression, the most interesting term is the first term as it is, in the limit of a low amplitude of the phase modulation $(\varphi(t)<<\pi)$, proportional to the phase shift $\varphi(t)$. This term only exists if its coefficient is significant, i.e. if $\gamma^{\prime \prime} \neq 0$. This means that in presence of a photorefractive grating in a pure diffusion regime, this term disappears and the only remaining term is the term in $\cos (\varphi(t))$. In this case we have for the modulated part :

$$
\Delta I_{d}(x, t)=e^{-\alpha x} I_{d}(0,0)\left(e^{\gamma^{\prime} x}-1\right) \varphi^{2}(t)
$$


We thus see that the photorefractive coupling is not optimum for the photorefractive beamsplitter as it does not allow for the coupling in quadrature that is best suited for a linear phase detection. This is easy to understand as a zero phase shift between illumination and space charge field gratings corresponds to no energy transfer between the beams. A phase shift introduced on one beam shifts the illumination grating towards the space charge field grating giving possibility for energy transfer with an amplitude proportional to the phase shift. Nevertheless a quadrature detection is not always necessary as shown in ref [21] for the case of high amplitude binary phase shift modulation scheme used in coherent communication.

One way to remove this drawback is to use a polarization sensitive set-up in order to mix the components in quadrature as proposed in Ref.[4]. Another way is to introduce on the signal beam, during the time of the measurement that is supposed to be short, an additional $\pi / 2$ phase shift (with for example an electrooptic modulator) that allows to mix the components in quadrature. We then have :

$$
\Delta I_{d}(x, t)=2 e^{-\alpha x} I_{d}(0,0)\left[\left(e^{\gamma^{\prime} x}-1\right) \cos \left(\varphi(t)+\frac{\pi}{2}\right)\right]=2 e^{-\alpha x} I_{d}(0,0)\left[\left(1-e^{\gamma^{\prime} x}\right) \sin (\varphi(t))\right]
$$

Finally, a last possibility is to find a material for which the index grating is not $\pi / 2$ shifted with the illumination grating. This is for example the case for photorefractive semiconductors with an externally applied electric field. In this case, we have $\gamma^{\prime \prime} \neq 0$ and the modulated part of the signal is dominated by $\sin (\varphi(t))$ (as we suppose $\varphi(t)<<\pi$ we have $\cos (\varphi(t)) \approx 1$ ) and we have (from $(27)$ ):

$$
I_{d}(x, t)=e^{-\alpha x} I_{d}(0,0)\left[e^{2 \gamma^{\prime} x}+2 e^{\gamma^{\prime} x} \sin \left(\gamma^{\prime \prime} x\right) \sin (\varphi(t))\right]
$$

An illustration is given in Fig.4 for the parameters of a CdTe:V sample [22]. With an applied electric field of $2 \mathrm{kV} . \mathrm{cm}^{-1}$, a grating vector $\mathrm{k}=1 \mu \mathrm{m}^{-1}$ and an illumination of $100 \mathrm{~mW} . \mathrm{cm}^{-2}$ (material parameters are given in Ref.[22]). We calculate an amplitude photorefractive gain $\gamma=(0.11-0.64 i) \mathrm{cm}^{-1}$ and a complex time constant $\tau_{0}=\tau_{r}-i \tau_{i}$ with $\tau_{r}=0.33 \mathrm{~ms}$ and $\tau_{i}=0.14 \mathrm{~ms}$. We introduce these parameters (with an absorption $\alpha=1 \mathrm{~cm}^{-1}$ and a crystal thickness of $1 \mathrm{~cm}$ ) in expressions (15) and (16), and we calculate the transmission of a phase modulated signal (with a sinusoidal phase modulation $\varphi(t)=0.1 \times \sin (2 \pi v t)$ of frequency $v=32 \mathrm{MHz}$ ). We see, in Fig.4, that the fast phase modulation is perfectly transformed into an intensity modulation of the transmitted beam as predicted by (31) for the regime of $1 / v<<\tau_{0}$. Coming back to the general case, we calculate the frequency response of the component for a sinusoidal phase shift $\varphi(t)=0.1 \times \sin (2 \pi v t)$ of frequency $v$. We see in Fig.5 the high band pass filtering properties of the photorefractive beamsplitter. The cut-off frequency is about $150 \mathrm{~Hz}$ (cut-off at $3 \mathrm{~dB}$ ).

\section{$\underline{\text { VI Conclusion }}$}


We have established an analytical model for two beam coupling energy transfer in presence of modulated beams for a crystal presenting absorption. We solved the two beam coupling differential equation using the Laplace transform method. The found solution allows to calculate the kinetics of the photorefractive effect in presence of absorption. It shows that in the low gain and low absorption regime, the response of the photorefractive effect is exponential with a time constant which is the space charge field time constant taken in the middle of the crystal. With this model, we can simulate the photorefractive beam splitter in crystals with low gain and an absorption even greater than the photorefractive gain, a situation which is common in systems developed in the infrared region with semiconductors.

We show that, taking into account the absorption or not, the photorefractive effect is not the best adapted for a linear detection of phase modulated signals. The $\pi / 2$ shift between illumination and index gratings has to be compensated, either by an external system, like adding an additional $\pi / 2$ phase shift on one of the beams, or by applying an external electric field in order to have a photorefractive effect governed by the drift regime. 


\section{$\underline{\text { References }}$}

[1] A.E. Siegman. Appl. Opt. 5, 1588 (1966).

[2] G. Hamel de Monchenault, J.P. Huignard. J. Appl. Phys. 63, 624 (1988).

[3] F.M. Davidson, L. Boutsikaris. Opt. Eng. 29, 369 (1990).

[4] R.K. Ing, J.P. Monchalin. Appl. Phys. Lett. 59, 3233, (1991).

[5] M. Cronin-Golomb. in Digest of Topical Meeting on Photorefractive Materials, Effects and Devices I (1987, Washington) Paper ThC5, 142.

[6] L. Boutsikaris, F.M. Davidson. Appl. Opt. 32, 1559 (1993).

[7] C.T. Field, F.M. Davidson. Appl. Opt. 32, 5285 (1993).

[8] D. J. Webb, L. Solymar. J. Opt. Soc. Am. B 8, 2204 (1991).

[9] M. Horowitz, D. Kliger, B.Fisher. J. Opt. Soc. Am. B 7, 2369 (1990).

[10] A. Blouin, J.P. Monchalin. Appl. Phys. Lett. 65, 932, (1994).

[11] A. Partovi, J. Millerd, E.M. Garmire, M. Ziari, W.H. Steier, S.B. Trivedi, M.B. Klein. Appl. Phys. Lett. 57, 846, (1990).

[12] P. Delaye, L.A. de Montmorillon, H.J. von Bardeleben, G. Roosen. Appl. Phys. Lett. 64, 2640 (1994).

[13] F.P. Strohkendl, J.M.C. Jonathan, R.W. Hellwarth. Opt. Lett. 11, 312 (1986).

[14] N.V. Kukhtarev, V.B. Markov, S.G. Odulov, M.S. Soskin, V.L. Vinetskii, Ferroelectrics 22, 949 (1979); 22, 961 (1979).

[15] G.C. Papen, B.E.A. Saleh, J.A. Tataronis. J. Opt. Soc. Am. B 5, 1763 (1988).

[16] G.C. Papen, B.E.A. Saleh, J.A. Tataronis. Opt. Lett. 14, 287 (1989).

[17] Formula (2.3.122), "Formulaire pour le calcul operationnel". V.A. Ditkin, A.P. Prudnikov. Masson, Paris.

[18] Handbook of mathematical functions. Ed. M. Abramowitz, I.A. Stegun. Dover Publications, New York.

[19] J.M. Heaton, L. Solymar. Optica Acta 32, 397 (1985).

[20] F. Davidson, L. Boutsikaris, M. Krainak, Opt. Lett. 13, 506 (1988).

[21] F. Davidson, C.T. Field, J. Lightwave tech. 12, 1207 (1994).

[22] Y. Belaud, P. Delaye, J.C. Launay, G. Roosen. Opt. Commun. 105, 204 (1994). 


\section{Figure Captions}

Figure 1: Photorefractive two beam coupling set-up. A pump beam $\mathrm{E}_{\mathrm{i}}$ and a time modulated signal beam $\mathrm{E}_{\mathrm{d}}$ propagates along $\mathrm{x}$ direction and interacts through a two beam coupling scheme, thus modifying the transmission of the signal beam.

Figure 2: Photorefractive build-up in the diffusion regime for different value of absorption of the crystal between 0 and $1 \mathrm{~cm}^{-1}$ (see text for calculation parameters). The values of the intensity at the crystal output are normalized to their steady state value. The signal beam intensity at the entrance of the crystal is the same for all the curves.

Figure 3: Photorefractive build-up in the diffusion regime for different values of the photorefractive gain between 0 and $2 \mathrm{~cm}^{-1}$. The value of the intensity at the crystal output are normalized to their steady state value. The absorption is $\alpha=1 \mathrm{~cm}^{-1}$ (other parameters are the same as in Fig.2). The probe intensity at the entrance of the crystal is the same for all the curves.

Figure 4 : Transmitted signal (straight line) through a photorefractive beamsplitter, as a function of time. The input signal beam is sinusoidally phase modulated $(\varphi(t)=0.1 \times \sin (2 \pi v t)$, dashed line) with an amplitude of $0.1 \mathrm{rad}$ and a frequency $v=32 \mathrm{MHz}$ (see text for calculation parameters). The probe intensity at the entrance of the crystal is $I_{d}(0,0)=1$.

Figure 5 : Frequency response of the photorefractive beamsplitter for a phase modulation $\varphi(t)=0.1 \times \sin (2 \pi v t)$ (same calculation parameters as for Fig.4). 


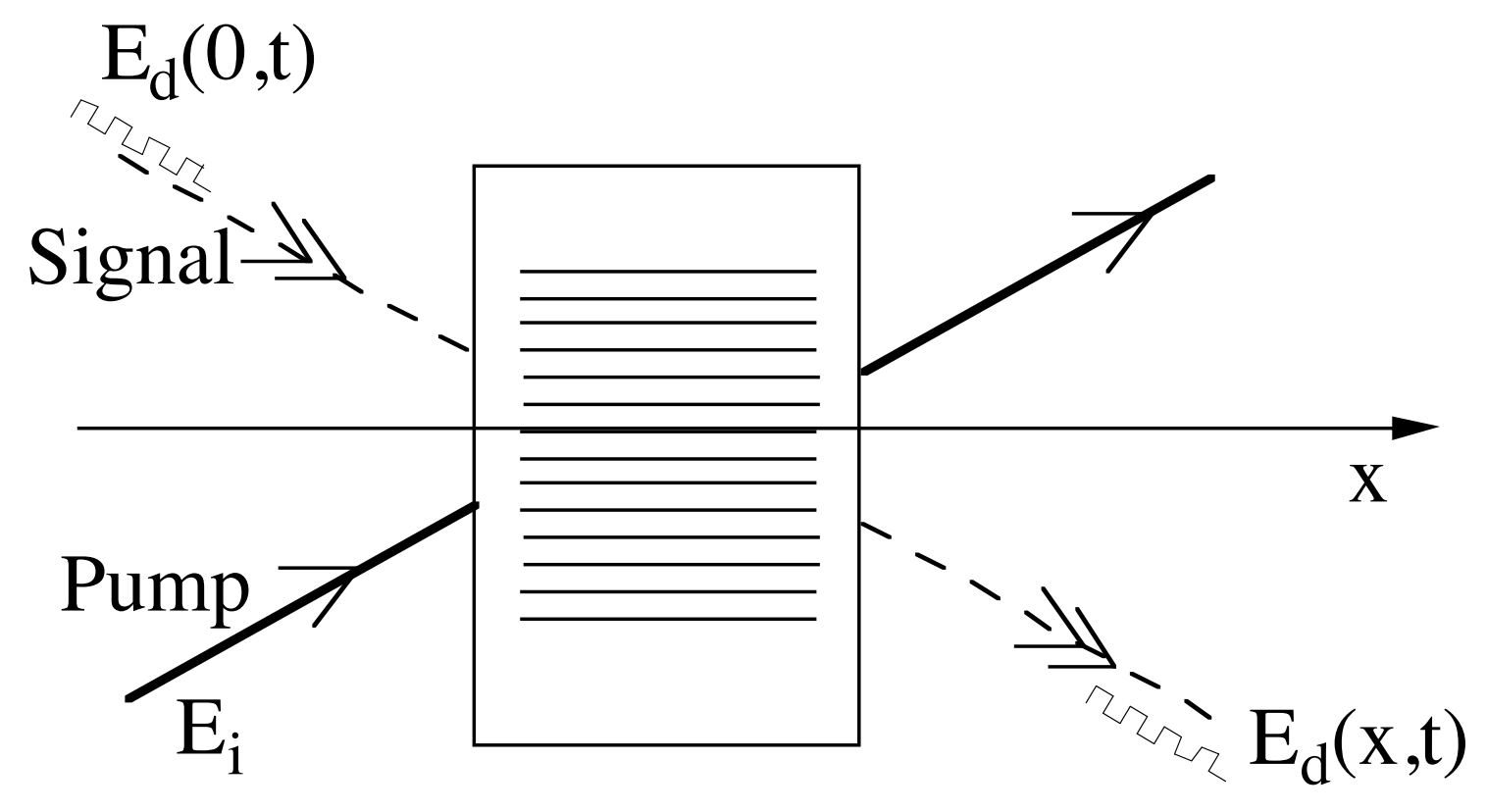

Figure 1 


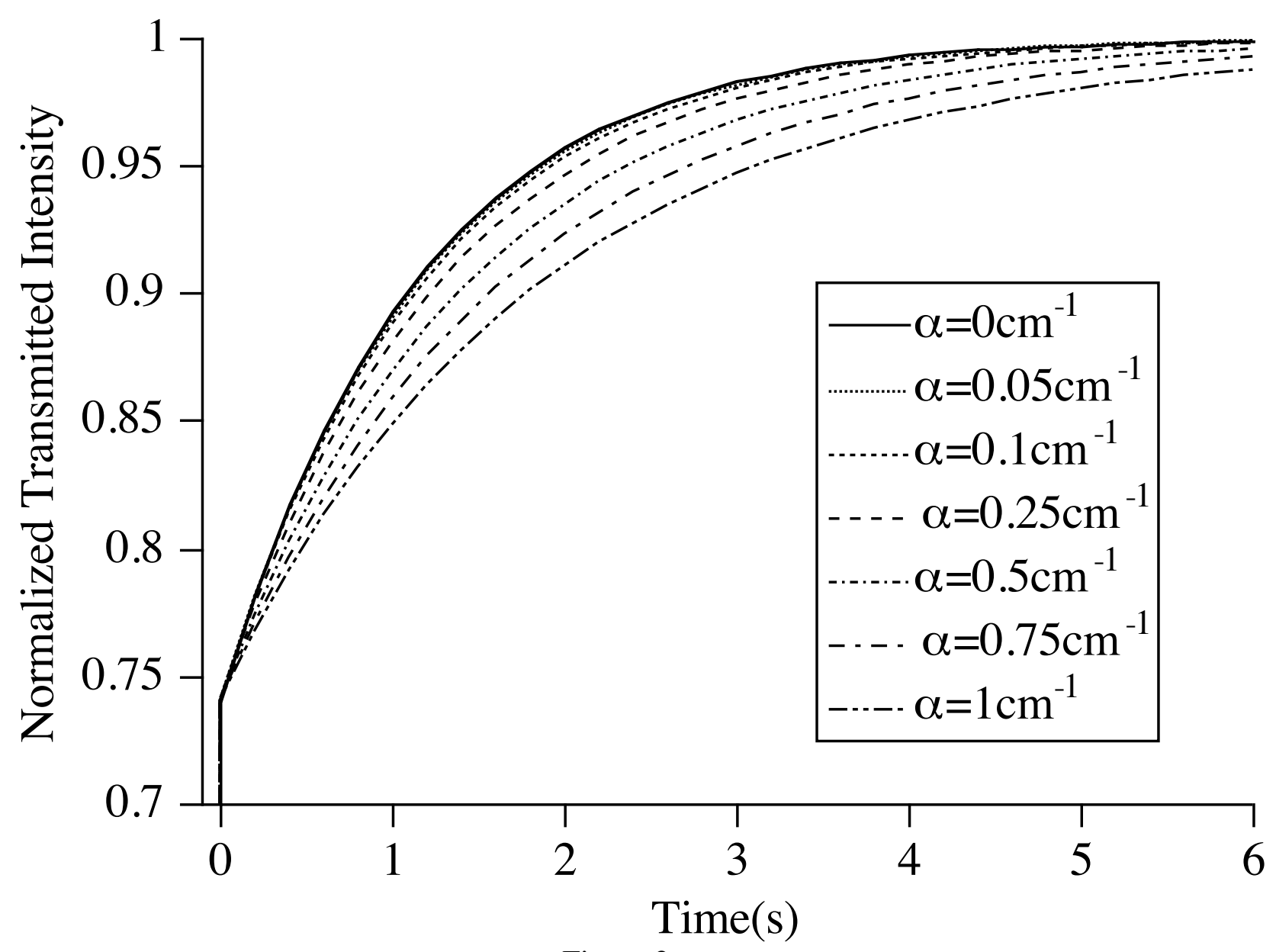

Figure 2 


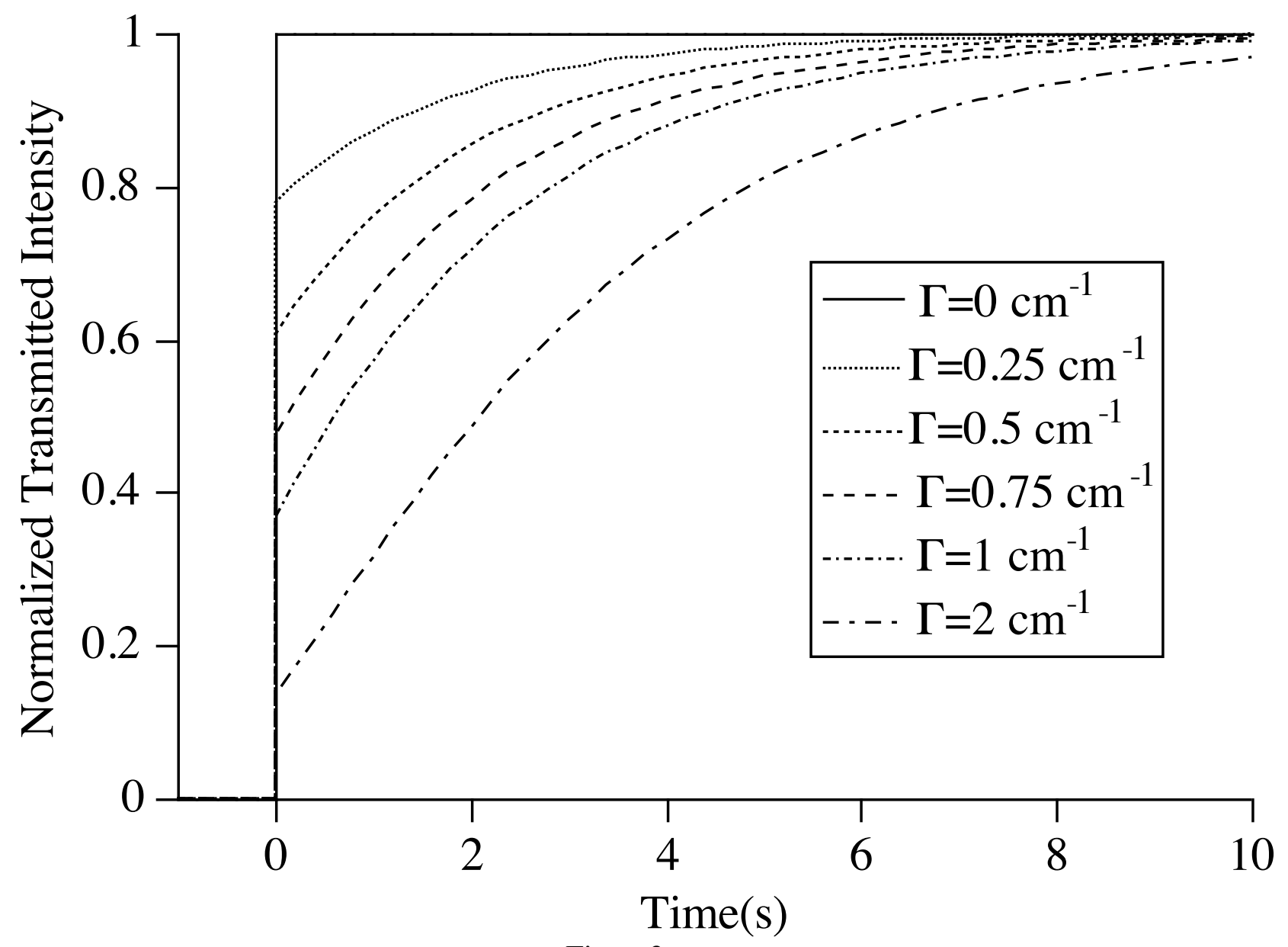

Figure3 


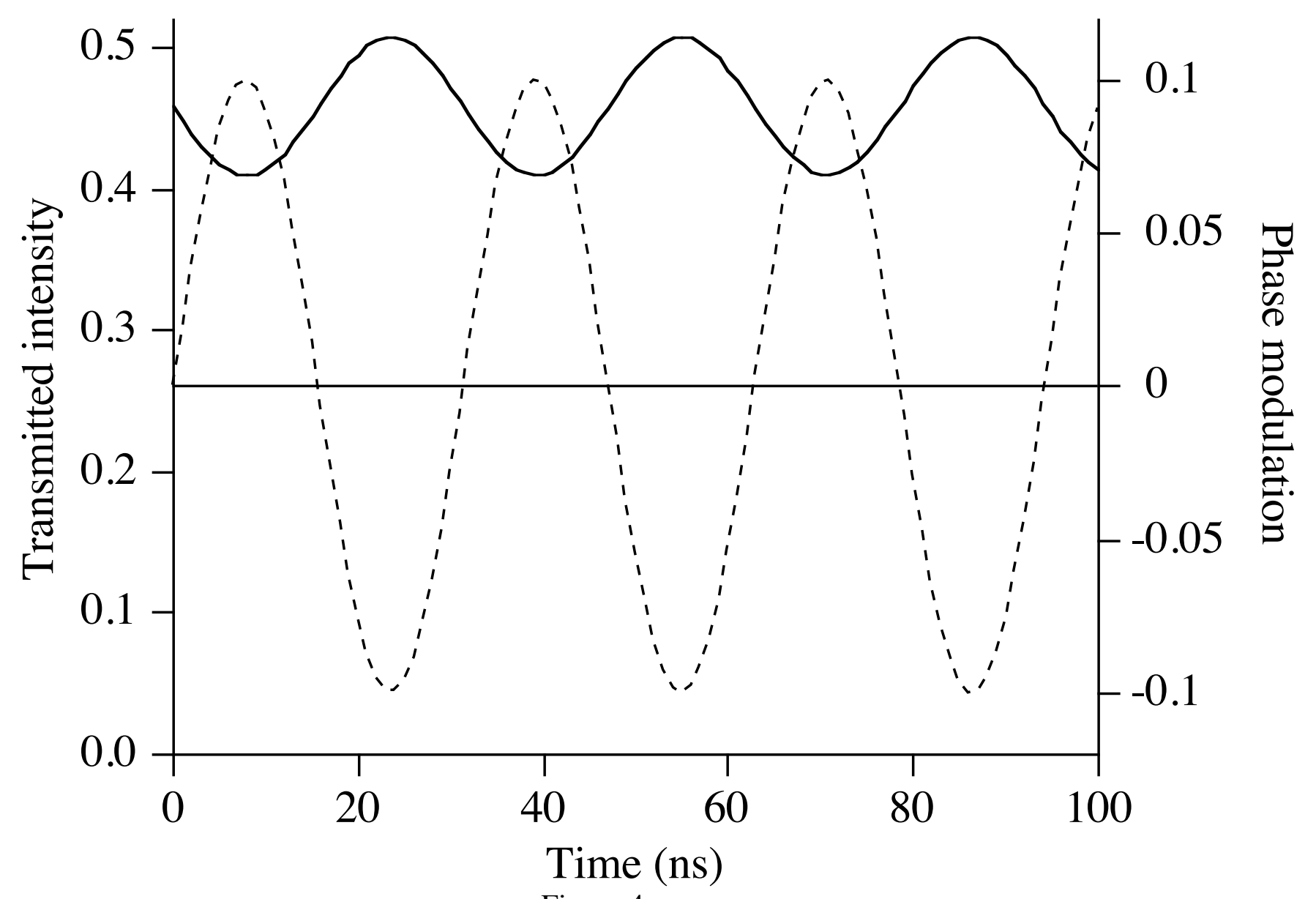

Figure 4 


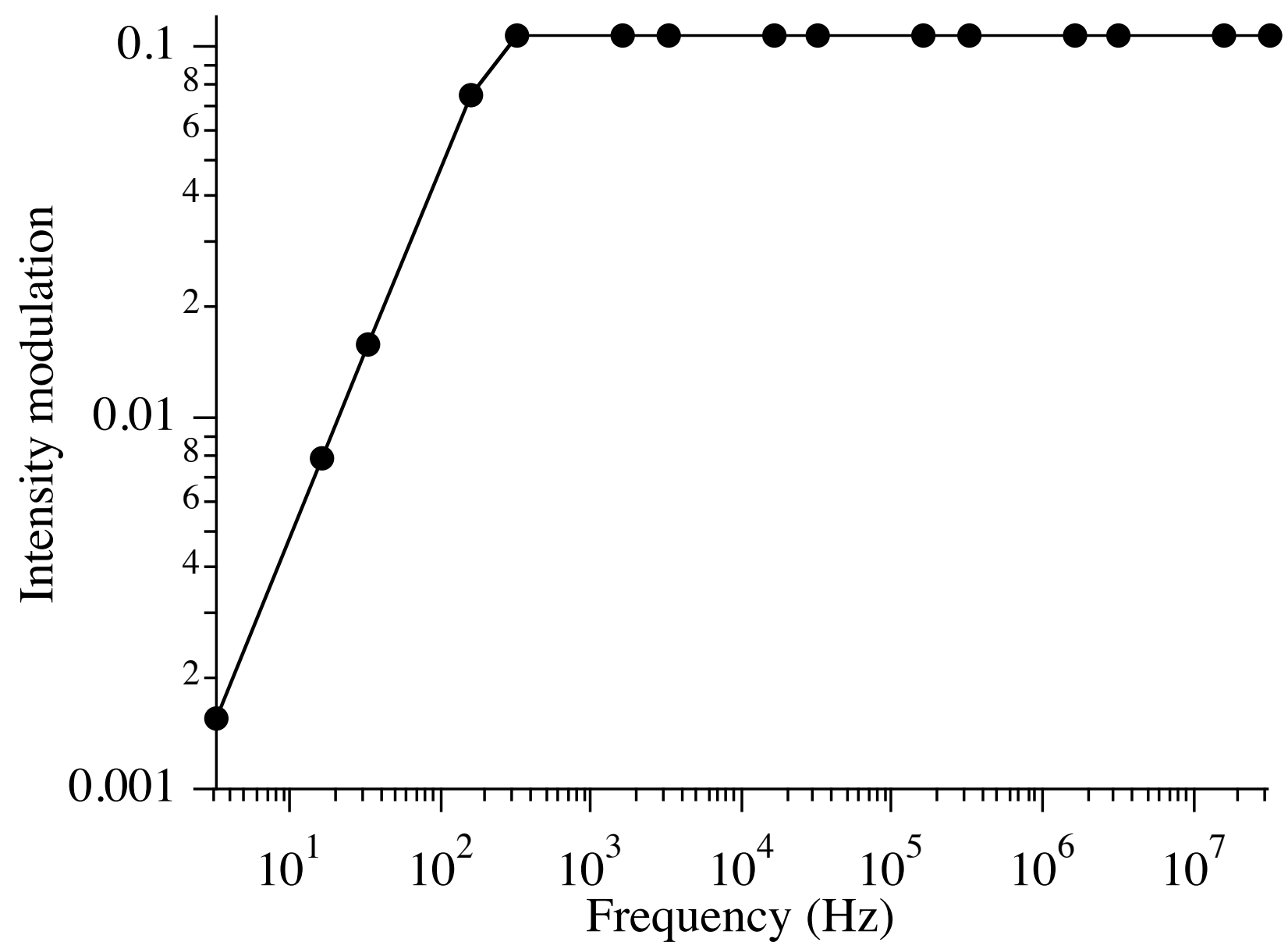

Figure 5 